\title{
Potential Molecular Mechanism of Rubi Fructus in the Treatment of T2DM Based on Multidimensional Biological Network
}

\author{
Wenjing Huang ${ }^{1,2}$, Jiana Hong ${ }^{2}$, Wenlu Chen ${ }^{3}$, Bingbing Li ${ }^{14^{*}}$, Shasha $\mathrm{Li}^{3}$, Guoliang Chen ${ }^{4}$, Xue Xiao ${ }^{2 *}$ \\ ${ }^{1}$ College of Traditional Chinese Medicine, Guangdong Pharmaceutical University, Guangzhou 510006, China \\ ${ }^{2}$ Institute of Chinese Medicine Sciences, Guangdong Pharmaceutical University, Guangzhou 510006, China \\ ${ }^{3}$ The Second Affiliated Hospital of Guangzhou University of Chinese Medicine, Guangzhou 510006, China \\ ${ }^{4}$ State Key Laboratory of Generic Manufacture Technology of Chinese Traditional Medicine, Lunan Pharmaceutical Group Co., Ltd., \\ Linyi, Shandong 276000, China
}

\begin{abstract}
Objective: To explore the potential targets and underlying mechanism of Rubi Fructus in the treatment of type 2 diabetes mellitus. Methods: Potential active components of Rubi Fructus were proposed by literature search and as well followed the Lipinski's Rule of Five. The therapeutic targets of potential active components and T2DM-related genes were selected through Swiss target prediction and Gene Cards. The network of potential active components and T2DM-related targets were constructed by Cytoscape 3.7.2, and STRING analysis was performed to reveal the interaction among target proteins and to build the network of protein-protein interaction. Both Gene ontology (GO) and Kyoto encyclopedia of genes and genomes (KEGG) enrichment analysis of core targets were performed using David database. Results: Totally, 68 potential active components of Rubi Fructus and 196 T2DM-related targets were found. Among them, 39 key targets were obtained by network analysis, which were primarily enriched on PI3K-Akt, HIF-1, TNF, Tolllike receptor, and Focal adhesion signaling pathways. Conclusion: For the treatment of T2DM, the active compounts of Rubi Fructus can regulate the expression of proinflammatory and oxidative stress mediators and improving insulin resistance through multiple components, targets, and pathways, which offers a scientific support to further Rubi Fructus studies and to the research on T2DM treatment.
\end{abstract}

\section{Introduction}

Rubi Fructus, the dried fruits of Rubus chingii $\mathrm{Hu}$, has the activities of ameliorating glycolipid metabolism disorder, anti-oxidation and anti-inflammation according to pharmacological studies ${ }^{[1-2]}$. Xie et al. discovered that raspberry ketone, a component of Rubi Fructus, was able to increase the expression of insulin in pancreatic $\beta$ cells among diabetic mice and showed a significant hypoglycemic effect ${ }^{[3]}$. T2DM is a chronic metabolic disease mainly featuring insulin resistance and it is usually induced by multiple risk factors such as gene, environment, and lifestyle, and it tends to lead to various complications like kidney injury, blood circulation disorder, and irregular heart function ${ }^{[4]}$. At present, chemical drugs are the dominant choice in the clinical treatment of T2DM. However, long-term medication can lead to a series of adverse reactions such as vomiting and ageustia, which has brought many troubles to patients' lives ${ }^{[5]}$. It has been a target for researchers to explore and develop a drug with minimal toxic and side effects. With multiple components like triterpenes, flavonoids, and phenolic acids, Rubi Fructus conceives abundant material basis and great potential in improving the glucose metabolic disorder ${ }^{[1,6]}$.

"Network pharmacology" is an effective technology to reveal the mechanism of drug action from the perspective of bioanalysis network based on the interaction network of "disease-genes-targets-drug", which demonstrates an evident resemblance to traditional Chinese medicines that feature the synergistic effect of multiple components, pathways, and targets ${ }^{[7-8]}$. With the purpose of offering a reference to future studies, the network pharmacology technique was employed to predict the medicinal material base, potential targets, and corresponding pathways of Rubi Fructus in the treatment of T2DM in this study.

\section{Materials and Methods}

\subsection{Software and Database}

Databases and software applied in this study included Pubchem (https://pubchem.ncbi.nlm.nih.gov/), Gene Cards (http://www.genecards.org/), Cytoscape 3.7.2, String11.0 (https://string db.org/), Interactivenn (http://www.interactivenn.net/), Swiss Target Prediction (http://www.swisstargetprediction.ch/), and DAVID 6.8 (https://david.ncifcrf.gov/).

\footnotetext{
*Corresponding author: erxiaohappy@163.com; libingt@126.com
} 


\subsection{Potential Active Components of Rubi Fructus and their Target Proteins}

The potential active Components of Rubi Fructus were collected by literature and Lipinski's Rule of Five. To be specific, the chemical components of Rubi Fructus were collected by literature ${ }^{[1,6]}$, the corresponding structural information, including molecular mass, water partition coefficient, the number of hydrogen-bonding acceptors and donors, and the number of rotatable bonds, were then searched in the database of Pubchem, and the potential active components of Rubi Fructus were eventually selected in accordance with Lipinski's Rule of Five.

Therapeutic targets of these potential active components were retrieved by inputting the SMILE strings of these components, into Swiss Target Prediction and setting "Homo sapiens" and "Probability $>0$ ".

\subsection{T2DM-related Targets Prediction}

T2DM-related targets were acquired by searching keywords "type 2 diabetes", "T2DM", and "diabetes mellitus type 2" in Gene Cards, a database that offers all gene information that has been known or is being explored

\subsection{Network Construction}

Therapeutic targets of potential active components of Rubi Fructus and T2DM-related targets were imported into the on-line drawing tool of Interactivenn so as to derive the Venn diagram, and the overlapped genes were deemed as the potential and T2DM-related targets of Rubi Fructus. Subsequently, the potential active components and potential targets of Rubi Fructus were input into Cytoscape3.7.2 to build the interaction network of potential active components of Rubi Fructus and potential targets of Rubi Fructus in the treatment of T2DM.

Besides, the potential targets were analyzed in String 11.0 to get the interactions between potential targets, and the results were exported in tsv format. Cytoscape 3.7.2 was utilized to draw the protein-protein interaction (PPI) network and a topological data analysis was performed through Network Analyzer to visualize important parameters among targets with the color depth of nodes standing for the Degree value and the thickness of lines among nodes referring to the Combined score. The core targets were further selected by setting the filter conditions of "simultaneously higher than the mean values of Degree, Betweenness Centrality, and Closeness Centrality”.

\subsection{Pathway Analysis of Core Targets}

In order to delineate the functions of biological processes and related signaling pathways, the GO and KEGG enrichment analysis were conducted by inputting the obtained core target genes of Rubi Fructus in the treatment of T2DM into the database of DAVID 6.8 and setting the $p$ value less than 0.01 .

\section{Results}

\subsection{Potential Active Components of Rubi Fructus and their Target Protein Prediction}

Totally, 68 potential active components of Rubi Fructus were obtained (Table 1) after literature search, and 650 therapeutic targets of these potential active components were identified by searching the target proteins of potential active components through the reverse docking in Swiss Target Prediction and removing the duplicated results.

Table 1. Potential Active Components of Rubi Fructus

\begin{tabular}{|c|c|}
\hline No. & Name \\
\hline F1 & ent-Labda-8(17),13E-diene-3 $\beta, 15,18$-triol \\
\hline $\mathrm{F} 2$ & ent-Labda-8(17),13E-diene-3 $\alpha, 15,18$-triol \\
\hline F3 & Kaempferol \\
\hline F4 & $\begin{array}{l}\text { Methyl 1-oxo-1,2-dihydroisoquinoline-4- } \\
\text { carboxylate }\end{array}$ \\
\hline F5 & $\begin{array}{l}\text { Methyl(3-hydroxy-2-oxo-2,3-dihydroindol-3-yl)- } \\
\text { acetate }\end{array}$ \\
\hline F6 & Linalyl acetate \\
\hline F7 & trans-Linalool oxide \\
\hline F8 & cis-Linalool oxide \\
\hline F9 & Terpineol-4 \\
\hline F10 & Calarene \\
\hline F11 & 3-Methyl-2-pentanone \\
\hline F12 & 1,1-diethoxyethane \\
\hline F13 & Isovaleraldehyde \\
\hline F14 & Ethyl acetate \\
\hline F15 & 2-Methylpentane \\
\hline F16 & 2-Heptanol \\
\hline F17 & Hexaldehyde \\
\hline F18 & 1-Hexene \\
\hline F19 & 1-Methyl-3-isopropylbenzene \\
\hline F20 & 1,2,3,5-Tetramethylbenzene \\
\hline F21 & Durene \\
\hline F22 & 3-Ethylstyrene \\
\hline F23 & 2,4-Dimethylstyrene \\
\hline F24 & 2,6-Dimethylcyclohexanol \\
\hline F25 & $\alpha$-Pinene \\
\hline F26 & Eucalyptol \\
\hline F27 & $p$-Cymene \\
\hline F28 & trans-Sabinene hydrate \\
\hline F29 & $\gamma$-Terpinene \\
\hline F30 & $\beta$-trans-Ocimene \\
\hline F31 & Methyl thymyl ether \\
\hline F32 & $\alpha$-Cedrene \\
\hline F33 & 4,7,9-Megastigmatrien-3-one \\
\hline F34 & Esculetin \\
\hline F35 & Imperatorin \\
\hline F36 & 4-Hydroxybenzoic acid \\
\hline F37 & Ellagic acid \\
\hline F38 & Ethyl gallate \\
\hline F39 & 4-Hydroxy-3-methoxy benzoic acid \\
\hline F40 & Gallic acid \\
\hline
\end{tabular}




\begin{tabular}{ll}
\hline F41 & Resveratrol \\
F42 & Vanillic acid \\
F43 & Raspberry ketone \\
F44 & Brevifolin carboxylic acid \\
F45 & $p$-Coumaric acid \\
F46 & Salicylic acid \\
& 4-[(2S,3R)-3-(Hydroxymethyl)-5-(3- \\
F47 & hydroxypropyl)-7-methoxy-2,3-dihydro-1- \\
& benzofuran-2-yl]-2-methoxyphenol \\
F48 & Ferulic acid \\
F49 & 4-Hydroxy-3-methoxybenzoic acid \\
F50 & Vanillin \\
F51 & 4-Hydroxyphenylacetic acid \\
F52 & Caproic acid \\
F53 & Caprylic acid \\
F54 & Oxalic acid \\
F55 & Tartaric acid \\
F56 & Malic acid \\
F57 & Citric acid \\
F58 & 2-Hydroxyquinoline-4-carboxylic acid \\
F59 & Shikimic acid \\
F60 & Phthalic acid \\
F61 & Mono- $n$-butyl phthalate \\
F62 & Ascorbic acid \\
F63 & Adenosine \\
F64 & Uridine \\
F65 & p-Hydroxybenzaldehyde \\
F66 & Di- $n$-butyl phthalate \\
\hline & \\
\hline
\end{tabular}

\begin{tabular}{ll} 
F67 & Ethylbenzene \\
F68 & 2-Butanone \\
\hline
\end{tabular}

\subsection{T2DM-related Targets Prediction and Potential Targets Identification}

A total of 11572 T2DM-related genes were retrieved in the database of Gene Cards by searching "type 2 diabetes", "T2DM", and "diabetes mellitus type", and the first 1000 genes were selected as the candidate T2DM targets in terms of "relevance score" ranking. The overlapped genes between the candidate T2DM targets and the therapeutic targets of components were identified through the Venn diagram, and 196 common targets were acquired and regarded as the potential targets of Rubi Fructus in the treatment of T2DM.

\subsection{Network Construction}

\subsubsection{Interaction Network of Potential Active Components of Rubi Fructus and Potential Targets of Rubi Fructus in the Treatment of T2DM}

Cytoscap3.7.2 was used to merge the network of active components targets and the PPI network, and the overlapped area was deemed as the active componentspotential targets network, as exhibited in Fig. 1.

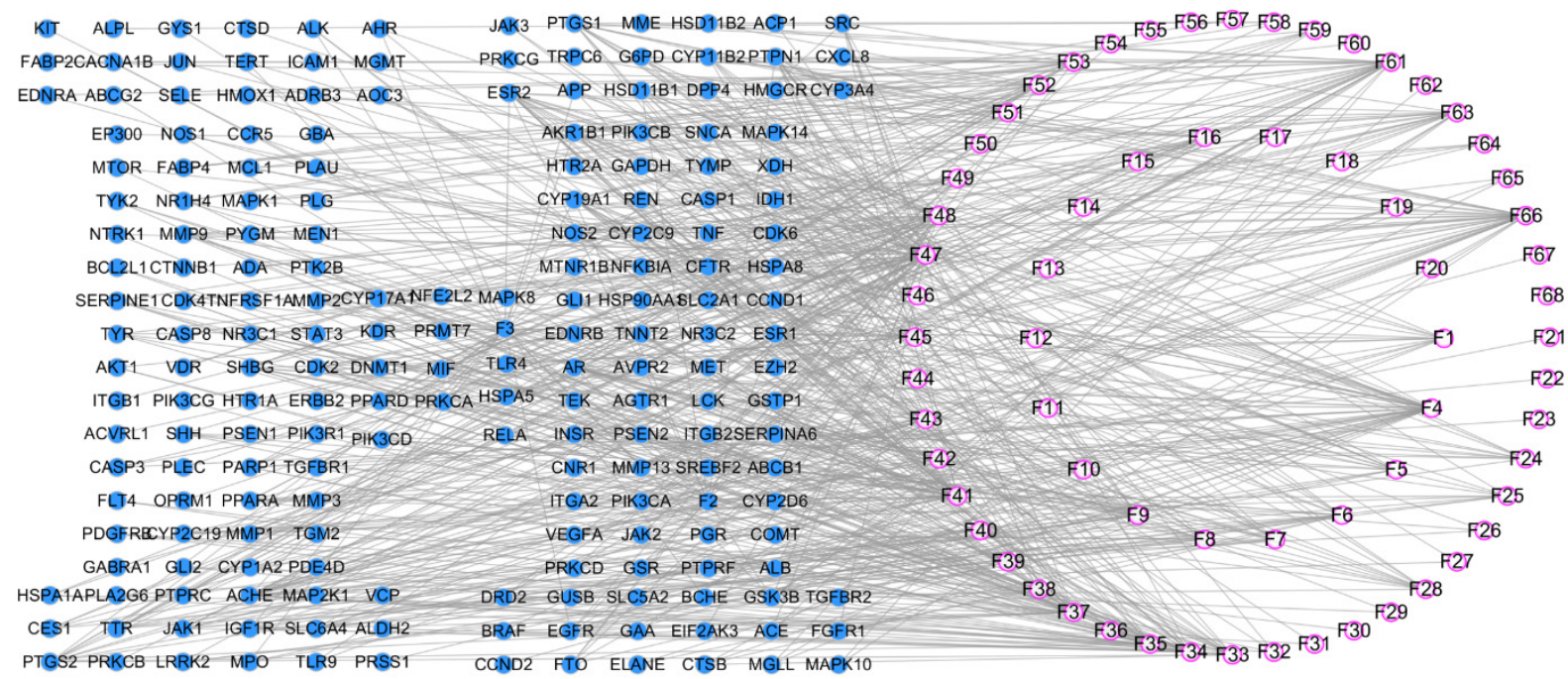

Fig. 1. Interaction Network of Potential Active Components of Rubi Fructus $(\bigcirc)$ and Potential Targets of Rubi Fructus $(\bigcirc)$ in the Treatment of T2DM

\subsubsection{Potential Target Protein Network of Rubi Fructus in the Treatment of T2DM}

The interaction between potential targets of Rubi Fructus in the treatment of T2DM was analyzed by importing the 196 potential targets derived in 2.2 into the database of String 11.0, and the result was exported in tsv format, which underwent a topological data analysis via
Cytoscape 3.7.2 (Fig. 2.). In the following figure, dots stood for targets with a darker color indicating a larger Degree value and more proteins related to the target, and lines referred to the interaction between targets with a thicker line suggesting a higher Combined score and stronger association with proteins. Core targets were searched and obtained under conditions " $>$ Degree average (36.4184), > Betweenness Centrality average(0.0046), $>$ Closeness Centrality average 
(0.5353)”, as shown in Table 2.

\subsection{GO Enrichment Analysis and KEGG Pathway Analysis}

GO enrichment analysis and KEGG pathway enrichment analysis were carried out by inputting 39 key targets into DAVID 6.8, and the first 15 biological processes and pathways were highlighted in the bubble diagram (Fig. 3) in terms of the number of involved targets. GO enrichment analysis $(P<0.01)$ demonstrated 34 biological processes such as the positive regulation of transcription from RNA polymerase II promoter, the positive regulation of nitric oxide biosynthetic process, glucose metabolic process, the regulation of inflammatory response,ect(Fig. 3-A). Targets were primarily enriched to 7 cellular components like nucleus, membrane raft, cytosol, extracellular space, ect(Fig. 3-B) and 5 molecular functions such as ATP binding, heme binding, nitric-oxide synthase regulator activity, ect(Fig. 3-C).

KEGG enrichment analysis revealed that 39 key targets were enriched into 84 signaling pathways, and most closely related to PI3K-Akt, HIF-1, TNF, Toll-like receptor, and Focal adhesion signaling pathway( Fig. 3-D)

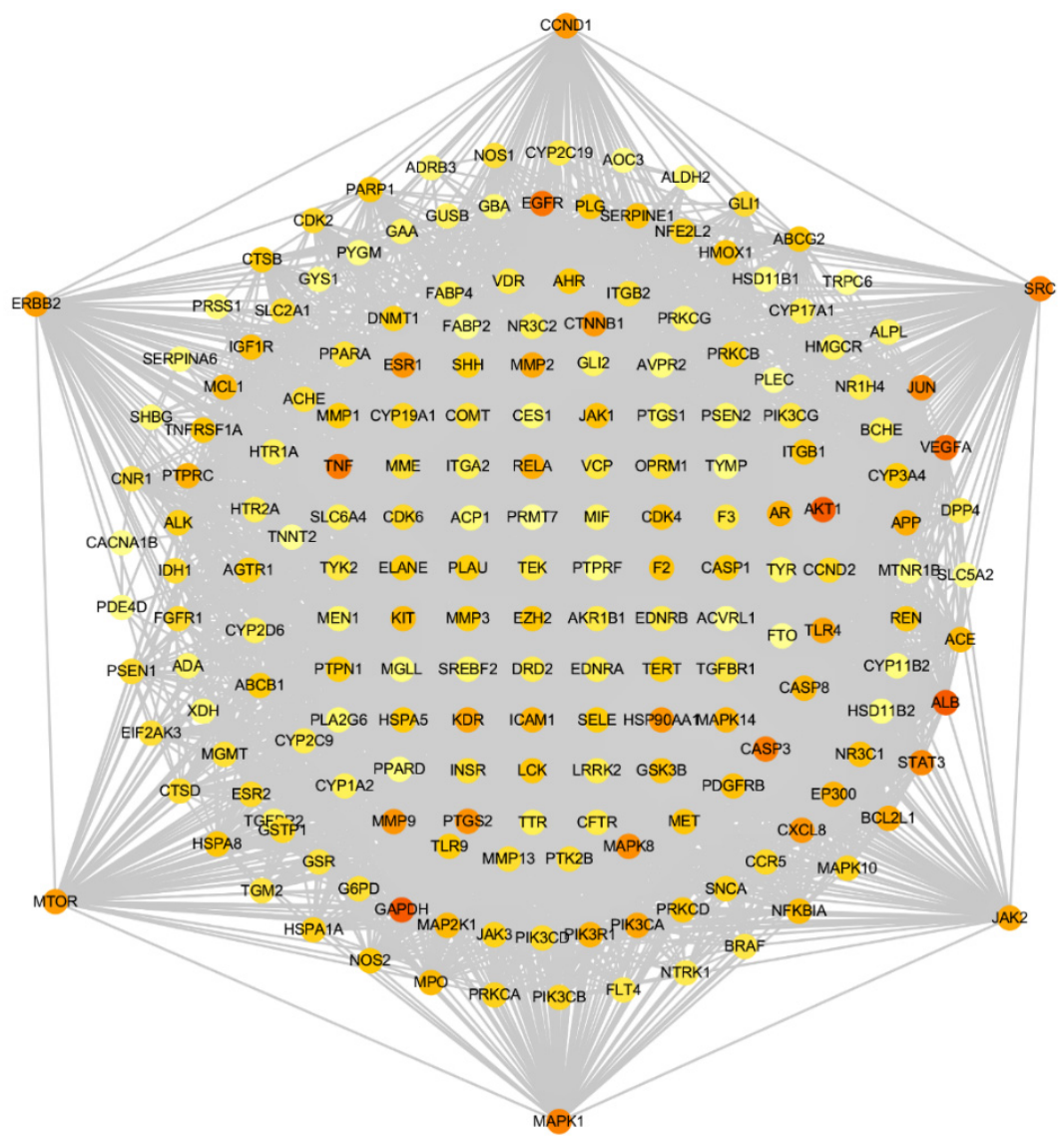

Fig. 2. Potential Target Protein Network of Rubi Fructus in the Treatment of T2DM

Table 2. Key Targets of Rubi Fructus in the Treatment of T2DM

\begin{tabular}{cccccccccc}
\hline No. & Gene & Degree & $\begin{array}{c}\text { Betweenness } \\
\text { Centrality }\end{array}$ & $\begin{array}{c}\text { Closeness } \\
\text { Centrality }\end{array}$ & No. & Gene & Degree & $\begin{array}{c}\text { Betweenness } \\
\text { Centrality }\end{array}$ & $\begin{array}{c}\text { Closeness } \\
\text { Centrality }\end{array}$ \\
\hline 1 & VEGFA & 119 & 0.0349 & 0.7169 & 21 & SRC & 103 & 0.0328 & 0.6771 \\
2 & KDR & 72 & 0.0122 & 0.6113 & 22 & MPO & 50 & 0.0056 & 0.5669 \\
3 & AKT1 & 135 & 0.0620 & 0.7647 & 23 & PTPRC & 60 & 0.0072 & 0.5838 \\
4 & TNF & 108 & 0.0235 & 0.6915 & 24 & F2 & 46 & 0.0073 & 0.5652 \\
5 & PIK3R1 & 60 & 0.0053 & 0.5821 & 25 & ERBB2 & 76 & 0.0068 & 0.6171 \\
6 & PIK3CA & 71 & 0.0078 & 0.6019 & 26 & EP300 & 61 & 0.0056 & 0.5909 \\
7 & CCND1 & 82 & 0.0075 & 0.6311 & 27 & MMP9 & 85 & 0.0174 & 0.6331 \\
8 & MAPK8 & 91 & 0.0103 & 0.6500 & 28 & ESR1 & 85 & 0.0138 & 0.6352 \\
9 & JUN & 95 & 0.0127 & 0.6588 & 29 & AR & 62 & 0.0059 & 0.5909 \\
10 & CASP8 & 56 & 0.0049 & 0.5752 & 30 & CXCL8 & 84 & 0.0168 & 0.6373 \\
11 & CASP3 & 106 & 0.0205 & 0.6866 & 31 & ITGB1 & 46 & 0.0055 & 0.5587 \\
12 & HSP90AA1 & 84 & 0.0151 & 0.6352 & 32 & ALB & 136 & 0.0845 & 0.7677 \\
\hline
\end{tabular}




\begin{tabular}{lccccccccc}
\hline 13 & MTOR & 77 & 0.0077 & 0.6210 & 33 & NR3C1 & 50 & 0.0145 & 0.5702 \\
14 & RELA & 64 & 0.0065 & 0.5963 & 34 & PTGS2 & 89 & 0.0168 & 0.6478 \\
15 & EGFR & 113 & 0.0300 & 0.7014 & 35 & CYP3A4 & 38 & 0.0073 & 0.5478 \\
16 & STAT3 & 94 & 0.0099 & 0.6566 & 36 & GAPDH & 137 & 0.0755 & 0.7677 \\
17 & APP & 64 & 0.0136 & 0.5945 & 37 & ACE & 52 & 0.0135 & 0.5718 \\
18 & CTNNB1 & 83 & 0.0143 & 0.6250 & 38 & REN & 42 & 0.0075 & 0.5571 \\
19 & JAK2 & 67 & 0.0048 & 0.5945 & 39 & TLR4 & 72 & 0.0076 & 0.6113 \\
20 & MAPK1 & 101 & 0.0275 & 0.6747 & & & & & \\
\hline
\end{tabular}

A

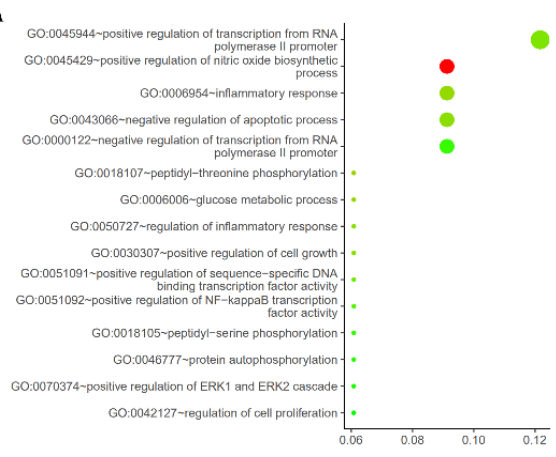

C

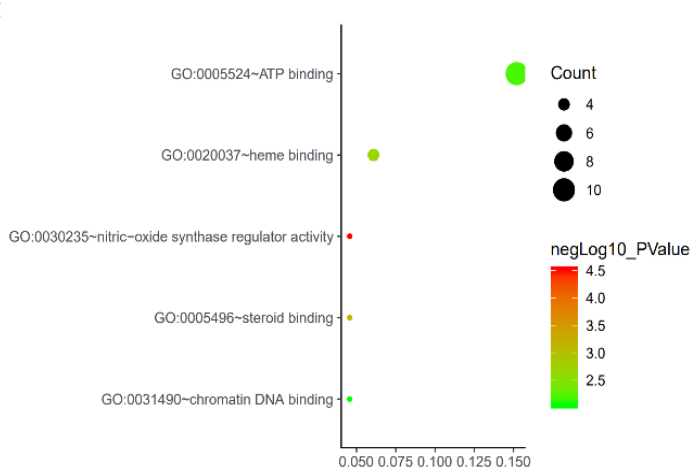

B

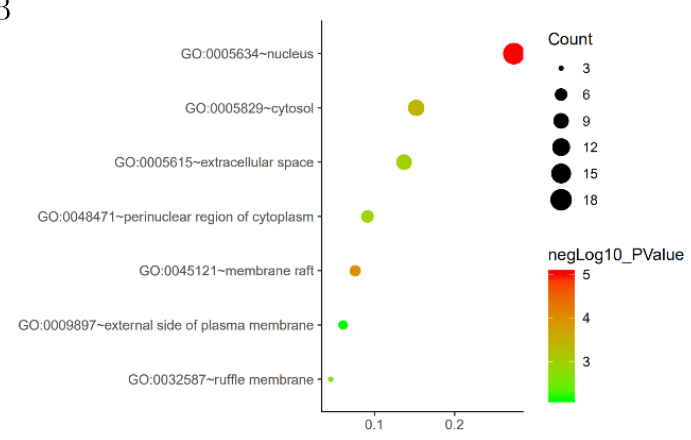

D)

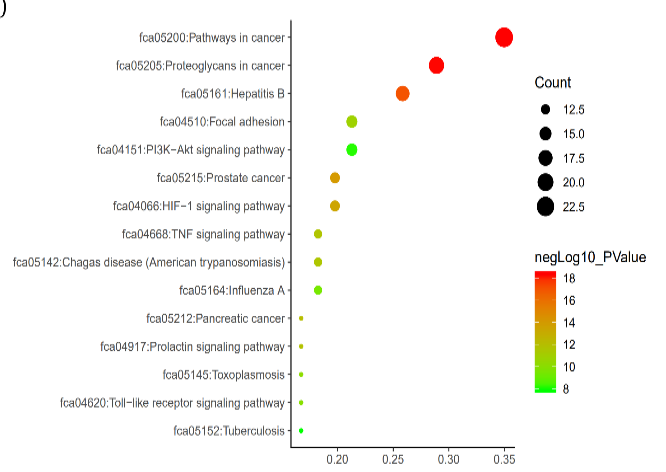

Fig. 3. Pathway Analysis of Rubi Fructus in the Treatment of Type 2 Diabetes Mellitus

\section{Discussion}

By adopting the "network pharmacology" technique based on a "holistic and systematic" concept, this study revealed the potential molecular mechanism of Rubi Fructus in the treatment of T2DM from the perspective of biological analysis network, hoping to offer a theoretical reference to future studies.

\subsection{Analysis of Potential Active Components of Rubi Fructus in the Treatment of T2DM}

The therapeutic effect of Rubi Fructus in treating T2DM and related complications is closely linked to its components, and this study discovered the dominant active components of Rubi Fructus might be kaempferol, ferulic acid, esculetin, ellagic acid, raspberry ketone, imperatorin, etc. Kaempferol is capable of regulating glycometabolism through AKT1 and EGFR. A previous study reported that kaempferol was able to alleviate the pathological injuries of kidney tissues among diabetic nephropathy rats, suppress inflammatory responses, reduce oxidative stress, and improve renal functions ${ }^{[9]}$. It's also found that kaempferol could treat diabetic retinopathy by inhibiting the activation of Src-Aktl-Erk 1/2 signaling pathway via AEGF and PGF ${ }^{[10]}$. Ellagic acid is equipped with pharmacologic actions like anti-oxidative, anti-inflammatory, and anti-tumor effects ${ }^{[11]}$, and this study also uncovered that ellagic acid might improve insulin resistance, play an anti-oxidative role, and mitigate the occurrence and development of T2DM by regulating PI3K-AKT and HIF-1 signaling pathways through AKT1, EGFR, and CCND1. Moreover, ferulic acid can improve the blood glucose level of T2DM mice and could play its role by regulating the signal transduction of HIF-1 signaling pathway via EGFR and STAT3 ${ }^{[12]}$. In addition. raspberry ketone, esculetin, imperatorin, and shikimic acid were also potential chemical components of Rubi Fructus in the improvement of glucose metabolic disorder.

\subsection{Key Targets of Rubi Fructus in the Treatment of T2DM}

Drug targets refer to the binding sites of drugs in the body, containing genetic locus, receptors, enzymes and other biomacromolecules, and drugs play a therapeutic role usually by binding to specific drug targets. Based on this study, multiple components of Rubi Fructus such as kaempferol, ferulic acid, ellagic acid, and raspberry 
ketone were could treat T2DM by interacting with different targets like AKT1, TNF, STAT3, and MAPK8.

Protein kinase B (PKB, also known as AKT) is a leading downstream effector molecule of the activation of phosphatidylinositol 3-kinase (PI3K), and the phosphorylation-activated AKT is able to promote glycogen synthesis and glucose transport, enhance the insulin sensitivity, and lower the blood glucose level, constituting a key molecule in the final action of insulin ${ }^{[13]}$. As one of the three closely related protein kinases (AKT1, AKT2, and AKT3), AKT1 can take part in biological processes like metabolic regulation, cell survival, and cell proliferation, and regulate the signal transduction of PI3K-AKT pathway. Sun et al. found that flavonoids in potentilla disclor bunge could increase the expression of related proteins in PI3K-Akt signaling pathway in liver tissues from T2DM $\mathrm{db} / \mathrm{db}$ mice, and remarkably improved glucose and lipid metabolism disorder and insulin resistance ${ }^{[14]}$.

Tumor necrosis factor (TNF) can obstacle the activation of PI3K, interfere with the insulin signal transduction ${ }^{[15]}$, and activate the large production of interleukin-6 (IL-6) from lymphocytes, thus inducing the downstream production of inflammatory factors and promoting the occurrence and development of diabetic complications ${ }^{[16]}$. It can be concluded that TNF serves as an important molecular basis in the occurrence and development of diabetes mellitus and its complications. Lou et al. held that the yam gruel could down-regulate the expression levels of TNF- $\alpha$, IL- 6 , and CRP in the serum of $\mathrm{T} 2 \mathrm{DM}$ rats, and decline the mRNA and protein expression of PEPCK and G6Pase in the hepatic tissue, thus reducing the gluconeogenesis in the liver ${ }^{[17]}$.

Signal transducer and activator of transcription 3 (STAT3) is initially identified as an acute-phase inflammatory response factor and involves in the activation of IL- 6 response in acute-phase inflammatory response. IL-6 can promote the up-regulation of $p$ STAT3/STAT3 and enhance insulin sensitivity ${ }^{[18]}$, and in pancreatic $\beta$ cells, IL-22 can activate the STAT3-mediated signaling pathway by binding to IL-22 receptor in order to promote the reproduction of pancreatic $\beta$ cells, inhibit the apoptosis, and alleviate the inflammatory response ${ }^{[19]}$. Therefore, STAT3 might be a new target to interfere with glucolipid metabolism-related diseases and the inflammatory response.

\subsection{KEGG Pathway Analysis}

After the binding between the drug and targets, cells will play their roles by receiving the signal while sending the signal to downstream genetic locus, which will induce the secretion of various effector molecules and initiate the complicated regulation in the body. Interaction of "drugtargets-disease" is in no way the "one-to-one" relationship, but a complex interaction network. Thus, the following part will focus on some signaling pathways closely related to the treatment of T2DM by Rubi Fructus.

\subsubsection{PI3K-Akt Signaling Pathway}

Previous studies indicated that the activation of c-Jun Nterminal kinase (JNK) could promote the phosphorylation of serine in insulin receptor substrate-1 (IRS-1) and further inhibit the phosphorylation of its tyrosine. In the meanwhile, PI3K and AKT would be disturbed, thus injuring pancreatic $\beta$ cells, increasing apoptosis, lowering the level of insulin secretion, and inducing an array of T2DM symptoms like elevated blood glucose level ${ }^{[20-22]}$. The regulation of PI3K-Akt signal transduction could improve the occurrence and development of T2DM, and some extracts of traditional Chinese medicine were able to activate the PI3K-Akt signal transduction pathway and ameliorate insulin resistance ${ }^{[23]}$. Results from this study suggested that components of Rubi Fructus such as kaempferol, esculetin, and ellagic acid could regulate the PI3K-AKT signal transduction through multiple targets like AKT1, EGFR, VEGFA, and CCND1, thus contributing to the treatment of T2DM.

\subsubsection{TNF Signaling Pathway}

The tumor necrosis factor superfamily (TNFSF) consists of 19 ligands and 29 receptors, which have highly varied roles in the body. Among others, tumor necrosis factor $\alpha$ (TNF- $\alpha$ ) is able to accelerate the phosphorylation of IRS1 serine residues and inhibit the phosphorylation of IRS-1 tyrosine by acting on tumor necrosis factor receptor (P60), which can impede the activation of PI3K and affect the insulin signal transduction ${ }^{[24]}$. Besides, TNF- $\alpha$ can also activate the extensive production of IL- 6 in lymphocytes, and the secretion of IL- 6 will induce extensive production of downstream inflammatory factors, leading to blood vessel damage and facilitating the occurrence and development of diabetic complications ${ }^{[25]}$. Thence, the regulation of TNF signal transduction can improve insulin resistance and diabetic complications. Shikimic acid in Rubi Fructus can regulate the signal transduction of TNF signaling pathway via TNF target to ameliorate insulin resistance and diabetic complications.

\subsubsection{Toll-like Receptor Signaling Pathway}

Toll-like receptors (TLRs) can recognize damaged tissues and activate inflammatory responses in the body. Through TLR/NF- $\kappa$ B signal transduction pathway, TLRs can induce the abnormal secretion of the nuclear factor kappa $\mathrm{B}(\mathrm{NF}-\kappa \mathrm{B})$, mitogen-activated protein kinases (MAPKs), and $\mathrm{JNK}$, setting off a radical cascade reaction, and the activation of these kinases will obstacle insulin signal transduction ${ }^{[26]}$. Components of Rubi Fructus such as kaempferol, ferulic acid, esculetin, ellagic acid, and imperatorin can suppress the activation of kinases like MAPKs and JNK via AKT1, MAPK8 and other targets, finally controlling the signal transduction of Toll-like receptors signaling pathway.

\subsubsection{HIF-1 Signaling Pathway}

Both the excessive production of reactive oxygen species 
(ROS) and the reduced capacity of the antioxidant enzyme can disturb the insulin signal transduction ${ }^{[27]}$, and hypoxia inducible factor (HIF) serves as a key factor in regulating the oxygen homeostasis ${ }^{[28]}$. Ellagic acid that is equipped with the anti-oxidative effect can ameliorate insulin resistance and contribute to the treatment of liver complications of T2DM by down-regulating the expression of HIT- $\alpha{ }^{[30]}$. Moreover, other components of Rubi Fructus like raspberry ketone, ferulic acid, and esculetin can also improve T2DM and its complications by affecting this signaling pathway through targets such as AKT1 and VEGFA.

\section{Conclusion}

In this study, the results reveals that multiple potential active components of Rubi Fructus act on the biological processes and the signal pathways of PI3K-Akt, HIF-1, TNF, and Toll-like receptor signaling pathways through targets, thus mitigating the inflammatory response and ameliorating insulin resistance in the treatment of T2DM. This study provides a relatively systematic reference for researches on Rubi Fructus in the prevention and treatment of glucose metabolic disorder, yet further investigations are still needed in terms of the mentioned components, targets, and pathways.

\section{References}

1. L. Cui, Z.Q. Zheng, Gen. J. Stomatology 7(1), 192196(2020).

2. C.R. Gong, F.Z. Sun, H. Tian, et al. Food Sci. 31(3),239-242(2010).

3. X.M. Xie, X.B. Pang, X.T. Li, Chin. Pharm. J. 47(23), 1899-1904(2012).

4. R.F. Hu, X.B. Sun. Chin. J. Nat. Med. 15(6), 436441(2017).

5. Nandipati K.C., Subramanian S., Agrawal D.K.. Mol. Cell Biochem 426(1), 27-45(2017).

6. G. Yu, Z. Luo, W. Wang, et al. Front. Pharmacol 10(10),799(2019).

7. Y. Chen, Z. Chen, Q. Guo, et al. J. Agric. Food Chem. 67(25), 7025-7039(2019).

8. H.B. Chen, H.G. Zhou, W.T. Li, et al. Chin. J. Tradit. Chin. Med. Pharm. 34(7), 2873-2876(2019).

9. H.Y. Wang, J.G. Zhao, Z.G. Wei, et al. Biomed Pharmacother. 118,109379(2019).

10. X.H. Xu, C. Zhao, Q. Peng, et al. Braz. J. Med. Biol. Res., 50(3), 5396-5402(2017).

11. Usta C., Ozdemir S., Schiariti M., Puddu PE. Int. J. Food Sci. Nutr.64(7), 907-913(2013).

12. Narasimhan A., Chinnaiyan M., Karundevi B.. Appl. Physiol. Nutr. Metab. 40(8), 769-806(2015).

13. Ganesan K., B. Xu. Molecules 24(14):2556 (2019) .

14. X.N. Kong, H.Y. Cui, H.L. Zhou. Chin. J. Exp. Tradit. Med. Form. 27(3), 78-84(2021).

15. Boller S., Joblin B.A., L. Xu, et al. Arch. Physiol.
Biochem 118(3), 148-155(2012).

16. Y.L. Wu. Chin. J. Health. Lab. Technol. 28(23), 28882890(2018).

17. Z.T. Luo, S.Q. Pang, J. Zhou, et al. J. Hunan Univ.Chin. Med. 41(1), 46-52(2021).

18. H.F. Liu, C.Y. Wei. Tianjin Med. J. 48(4), 343347(2020).

19. X.T. Wang, Ota Naruhisa, Manzanillo Paolo, et al. Nat. 514(7521), 237-241(2014).

20. B. Dai, Q. Wu, C. Zeng, et al. J. Ethnopharmacol. 192,382-389(2016).

21. R. Fan, X. Li, X. Gu, et al. Diabetes Obes. Metab. 12(9), 815-824(2010).

22. Fan Y., He Z., Wang W., et al. Biomed Pharmacother, 106,733-737(2018).

23. J. Wang, Y. He, D. Yu, et al. Food Chem. Toxicol. 135,110965(2020).

24. X. Zhang, J.G. Chen, M. Wu, et al. Chin. J. Gen. Practice. 14(7), 1095-1097(2016).

25. F. Xu, X.L. Huang, H.M. Wu, et al. Chin. Tradit. Herbal Drugs 50(16), 3880-3890(2019).

26. X. Zhan, L. Wang, Z. Wang, et al. J. Clin. Biochem. Nutr. 64(3), 194-200(2019).

27. Annunziata G., Barrea L., Ciampaglia R., et al. Phytother Res. 33(9), 2213-2220(2019).

28. D. He, J.H. Huang, Z.Y. Zhang, et al. Drug Des. Devel. Ther. 13,3989-4005(2019).

29. D. Cheng, J. Li, B. Zhou, et al. Chin Med Mat. 35 (11), 1873-1876(2012).

30. Koyasu S., Kobayashi M., Goto Y., et al. Cancer Sci. 109(3), 560-571(2018).

31. Reusch J.E., Manson J.E.. JAMA. 317(10), 10151016(2017).

32. X. Yin, Z. Xu, Zhang Z., et al. Diabetes Res. Clin. Pract. 128,127-135(2017). 